\title{
Determinants of multidrug-resistant tuberculosis in Addis Ababa, Ethiopia
}

\section{Demelash Assefa \\ Berhanu Seyoum ${ }^{2}$ \\ Lemessa Oljira ${ }^{2}$}

'KNCV Tuberculosis Foundation, Addis Ababa, ${ }^{2}$ School of Public Health, College of Health and Medical Sciences, Haramaya University, Harar, Ethiopia
Correspondence: Berhanu Seyoum School of Public Health, College of Health and Medical Sciences, Haramaya University, P. O. Box: 235, Harar, Ethiopia

Tel +25 I 91 1677774

Email seyoumbe07@gmail.com
This article was published in the following Dove Press journal:

Infection and Drug Resistance

II July 2017

Number of times this article has been viewed
Abstract: Multidrug-resistant tuberculosis (MDR-TB) has been jeopardizing the global effort to prevent and control tuberculosis (TB). However, data on MDR-TB in Ethiopia in general, and in our study area in particular, are very scarce. The aim of this study was to identify determinants of MDR-TB in a cohort of patients managed in two referral hospitals of Addis Ababa, Ethiopia, between April 1, 2013 and June 30, 2013. A health facility-based case-control study was conducted. A total of 710 TB patients (229 confirmed MDR-TB and 481 drug susceptible TB patients) were enrolled in this study. Identified independent determinants for MDR-TB were contact history with a known TB patient (adjusted odds ratio [AOR]: 1.9, 95\% CI: 1.1-3.3), previous history of TB treatment (AOR: $11.9,95 \% \mathrm{CI}: 6.8-21$ ), history of hospitalization (AOR: 4.4 95\% CI: 2.2-7.8), sputum-smear positivity (AOR: 1.9, 95\% CI: 1.1-3.4), and social stigma (AOR: 5.1, 95\% CI: 1.8-14.4). These identified factors should be considered for use in MDR-TB screening tool development if universal drug susceptibility testing is not possible. Moreover, TB infection control practices in health care settings should be further strengthened. As factors may vary from region to region, further broader studies need to be conducted in other parts of Ethiopia. Keywords: MDR-TB, drug susceptible TB, determinants, sputum smear positive, treatment completed, cured, drug susceptibility testing

\section{Introduction}

Ethiopia is one of the 27 high burden multidrug-resistant tuberculosis (MDR-TB) countries identified by the World Health Organization (WHO). According to the 2005 nationwide anti-tuberculosis drug resistance survey report, among 804 newly diagnosed tuberculosis (TB) cases, 1.6\% were infected with MDR-TB strains. The same report showed that out of 76 previously treated TB cases, $11.8 \%$ were developed MDR-TB. ${ }^{1}$ WHO estimated that 5825 MDR-TB cases existed in Ethiopia in the year 2010. As a result, the country has planned to treat $>8500$ MDR-TB patients until $2015 .^{2}$

Treatment of MDR-TB (Mycobacterium tuberculosis strains resistant to at least isoniazid and rifampicin [Sigma-Aldrich Co., St Louis, MO, USA]) is very complex. The treatment is given at least for 2 years. The drugs are more toxic and expensive than those used to treat patients without MDR-TB. ${ }^{3-5}$ As a result, new TB drugs are in pipeline in order to shorten, simplify and make MDR-TB treatment more effective. Moreover, public health strategies have been developed to prevent the occurrence of drug resistance, which usually results from human mistakes. ${ }^{2,6}$

Application of the traditional approach of National TB Program focusing on TB control per the WHO Stop TB Strategy, which is replaced by the new Post-2015 Strategy, focused on the concept of TB elimination (i.e., $<10$ new smear positive TB cases 
per 100,000 population) allows to prevent the occurrence of drug resistance in a cost-effective manner., ${ }^{1,7}$

To reduce the burden of MDR-TB, Ethiopia has designed a strategy to provide culture and drug susceptibility testing (DST) services at least to all MDR-TB suspected cases. ${ }^{8}$ However, there are limited data on the determinants of MDR-TB in the country to guide the National TB Program in designing an evidence-based algorithm to prioritize access to MDR-TB diagnostic implemented services (culture and DST) and tailor prevention and control strategies.

Therefore, this study was conducted to identify determinants of MDR-TB in a cohort of patients managed in referral hospitals of Addis Ababa, Ethiopia, between April 1, 2013 and June 30, 2013.

\section{Methods}

Health facility-based case-control study design was employed. Cases were all culture confirmed MDR-TB patients referred from all locations of Addis Ababa and registered at St. Peter TB Specialized and All Africa Leprosy, Tuberculosis and Rehabilitation Training Centre (ALERT) Hospitals, which are the only 2 centers in Addis Ababa mandated to provide second-line anti-TB treatment during the study period.

Controls were all consecutive pulmonary TB patients whose last first-line anti-TB treatment outcome was cured or treatment completed regardless of their previous history of TB treatment. They were selected from 11 randomly selected health facilities in Addis Ababa from where MDRTB cases were diagnosed and referred to the second-line treatment centers. Children aged $<5$ years old, and patients whose permanent residence was out of Addis Ababa were excluded from the study.

A double population proportion formula was used to calculate the sample size, considering 95\% two-sided confidence level, $80 \%$ power and 1:2 case to control ratio. Finally, a total of 229 cases and 481 controls were included in this study.

Data collection was carried out using pre-tested structured questionnaires. The questionnaires were filled by trained data collectors through interviews with cases and controls. To maintain data validity, regular supervision was carried out by trained supervisors and investigators. Cases were interviewed during their follow-up visit to the hospital or during hospital admission, and controls at completion of their treatment (when declared as treatment completed or cured). After completing the interview section, additional data were collected from the MDR-TB registers and Drug Susceptible (DS)-TB registers as per the protocol.
Outcome variables were MDR-TB or DS-TB status. Whereas, independent variables include age, sex, marital status, educational status, TB contact history, TB disclosure status, history of imprisonment, history of previous TB treatment, history of hospitalization, cigarette smoking habits, alcohol drinking habits, HIV status, presence of diabetes, history of chronic obstructive pulmonary disease (COPD) and psychiatric disorders.

\section{Data analysis}

Data were cleaned and entered into Epi data version 3.02 and analyzed using SPSS version 16.0. Cross tabulation was done to describe the demographic characteristics of cases and controls. Bivariate analysis was done for each variable to assess possible association(s). Variables with $P<0.25$ at bivariate analysis were entered into multivariate logistic regression model and adjusted odds ratio (AOR) with 95\% $\mathrm{CI}$ were calculated to determine the independent association between independent and outcome variables. $P$ value $<0.05$ was considered statistically significant.

\section{Ethical considerations}

The study was reviewed and approved by the Institutional Research Ethical Review Committee (IRERC) of Haramaya University, College of Health and Medical Sciences, the Research Ethical Review Committee of Addis Ababa City Administration Health Bureau, St. Peter TB Specialized Hospital and the ALERT Hospital. Informed consent was obtained from each study participant before initiation of data collection. All patients information were coded and kept confidential (no names and personal identifiers were used during data collection). Patients were duly informed of their right to withdraw from the study at any time and assured that, this will not affect the standard of health care provided to them.

\section{Results}

\section{Description of study population}

A total of 710 patients (229 confirmed MDR-TB cases and 481 DS-TB controls) were enrolled in this study. More male participants were found among controls $(57.8 \%)$ than among cases (47.6\%) ( $P$ value: 0.014$)$. About $92 \%$ of the cases and $80 \%$ of the controls were $<44$ years old $(P<0.001)$. Nearly $51 \%$ of the controls and $66 \%$ of the cases were single $(P<0.001)$. Overall, $22 \%$ of the patients had smoking habits (previously and/or currently), (18.3\% of the cases and $23.3 \%$ of the controls; $P$ value: 0.162 ). The proportion of previous alcohol consumers among the cases and the controls were $23 \%$ and $29 \%$, respectively ( $P$ value: 0.14 ). A total of $39.7 \%$ 
of the cases and $25.6 \%$ of controls responded that they had contact with a known TB patient at home or outside $(P<0.001)$ (Table 1).

\section{Description of independent determinants for MDR-TB}

Multivariate analysis revealed that patients who had contact history with known TB patient were twice higher among the cases than the controls (AOR: 1.9; 95\% CI: 1.1-3.3). History of previous TB treatment had strong association with MDRTB (AOR: 11.9, 95\% CI: 6.8-21). Patients who had been hospitalized for TB management were 4 times more likely to have MDR-TB than drug susceptible TB (AOR: 4.4; 95\% CI: 2.2-7.8). The current study also identified that probability of sputum-smear positivity was found to be higher in the cases than the control (AOR: 1.9, 95\% CI: 1.1-3.4).

There was a significant association between social stigma and development of MDR-TB (AOR: 5.2; 95\% CI: 1.8-14.4). Patients who were co-infected with HIV and co-morbid with

Table I Description of Sociodemographic characteristics of study participants in Addis Ababa, Ethiopia, between April 2013 and June 2013

\begin{tabular}{|c|c|c|c|c|c|}
\hline \multirow[t]{2}{*}{ Variables } & \multicolumn{2}{|c|}{$\begin{array}{l}\text { Case } \\
(N=229)\end{array}$} & \multicolumn{2}{|c|}{$\begin{array}{l}\text { Control } \\
(\mathrm{N}=48 \mathrm{I})\end{array}$} & \multirow[t]{2}{*}{$P$-value } \\
\hline & $\mathbf{N}$ & $\%$ & $\mathbf{N}$ & $\%$ & \\
\hline \multicolumn{6}{|l|}{ Sex } \\
\hline Male & 109 & 47.6 & 278 & 57.8 & 0.014 \\
\hline Female & 120 & 52.4 & 203 & 42.2 & \\
\hline \multicolumn{6}{|l|}{ Age (in years) } \\
\hline $0-44$ & 210 & 91.7 & 385 & 80.0 & 0.001 \\
\hline$\geq 45$ & 19 & 8.3 & 96 & 20.0 & \\
\hline \multicolumn{6}{|l|}{ Mean age $=31.7$ years } \\
\hline \multicolumn{6}{|l|}{ Marital status } \\
\hline Single & 152 & 66.4 & 243 & 50.5 & 0.001 \\
\hline Married & 64 & 27.9 & 179 & 37.2 & \\
\hline Divorced and widowed & 13 & 5.7 & 59 & 12.3 & \\
\hline \multicolumn{6}{|l|}{ Educational status } \\
\hline Illiterate, and read and write & 31 & 13.5 & 74 & 15.4 & 0.001 \\
\hline I-8 grade & 65 & 28.4 & 174 & 36.2 & \\
\hline $9-12$ grade & 7I & 31 & 165 & 34.3 & \\
\hline$\geq 12$ grade & 62 & 27.1 & 68 & 14.1 & \\
\hline \multicolumn{6}{|l|}{ Smoking habit } \\
\hline Yes & 42 & 18.3 & 112 & 23.3 & 0.162 \\
\hline No & 187 & 81.7 & 369 & 76.7 & \\
\hline \multicolumn{6}{|l|}{ Alcohol drinking habit } \\
\hline Yes & 53 & 23.1 & 138 & 28.7 & 0.142 \\
\hline No & 176 & 76.9 & 343 & 71.3 & \\
\hline \multicolumn{6}{|l|}{ HIV status of the patient } \\
\hline Unknown & 8 & 3.5 & 21 & 4.4 & 0.078 \\
\hline $\mathrm{R}$ & 37 & 16.2 & III & 23.1 & \\
\hline NR & 184 & 80.3 & 349 & 72.6 & \\
\hline
\end{tabular}

Abbreviations: HIV, human immunodeficiency virus; R, reactive; NR, non-reactive. diabetes were less likely to have MDR-TB than DS-TB (AOR: 0.3 ; 95\% CI: $0.2-0.7$ and AOR: 0.2 ; 95\% CI: $0.05-0.8$, respectively) (Table 2).

\section{Discussion}

This study revealed that those patients who had contact with known TB patients were about 2 times more likely to develop MDR-TB than DS-TB. Similar findings have been observed in Peru ${ }^{9}$ and in Europe. ${ }^{10}$ This can be explained by delays in diagnosis and treatment of MDR-TB patients than DS-TB patients, especially in low-income countries due to sub-optimal availability of diagnostic and treatment services coupled with poor infection prevention measures, both at home and at the health care facility level. These circumstances increase the duration of infectious period while producing additional infections from which future cases of MDR-TB will develop.

Having history of previous TB treatment was about 12 times more common among cases compared to controls (AOR: 11.9). This finding was consistent with studies conducted elsewhere, ranging from $30 \%$ to $80 \% .{ }^{11-14}$ In Ethiopia, the prevalence of MDR-TB among previously treated cases $(11.8 \%)$ is higher than among new cases $(1.6 \%) .{ }^{8}$ As in other high TB-incidence setting, most MDR-TB patients initially undergo first-line TB treatment till they are considered treatment failures and have access to MDR-TB diagnostic services.

Having sputum smear positive pulmonary TB was about 2 times more common among cases than controls (AOR: 1.9), testifying that a proportion of these cases is diagnosed at an advanced stage of the disease.

In this study, history of hospitalization for TB management was also an independent determinant for development of MDR-TB (AOR: 4.4). Similar findings were reported in South African studies (AOR: 3.8 and 2.08), ${ }^{11-14}$ which implies that hospital-acquired transmission of MDR-TB strains is more common.

In the current study, stigma was indirectly assessed using the patient's disclosure status of his/her TB disease at diagnosis. Patients who did not disclose their TB disease had, in fact, a greater probability for developing MDR-TB (AOR: 5.2). A report in Nepal indicated that stigma has been an independent predictor for MDR-TB (OR=2.65). ${ }^{13}$

Patients who feared social stigma are more likely to lack support from their family, friends or colleagues. Furthermore, the difficulty to access sick leaves from their employer and the related possible difficulty to take the prescribed medicines regularly might play a role. 
Table 2 Multiple logistics regression analysis of determinants for MDR-TB in Addis Ababa, Ethiopia, between April 2013 and June 2013

\begin{tabular}{|c|c|c|c|}
\hline Variables & COR (95\% Cl) & $P$-value & AOR $(95 \% \mathrm{Cl})$ \\
\hline \multicolumn{4}{|c|}{ Educational status } \\
\hline Illiterate & 1.00 & & 1.00 \\
\hline I-8 grade & $0.89(0.54-1.48)$ & 0.66 & $0.75(0.32-1.74)$ \\
\hline $9-12$ grade & $1.03(0.62-1.69)$ & 0.92 & $0.60(0.26-1.42)$ \\
\hline$>12$ grade & $2.18(1.27-3.74)$ & 0.005 & $2.04(0.85-4.89)$ \\
\hline \multicolumn{4}{|c|}{ Known TB contact history } \\
\hline No & 1.00 & & 1.00 \\
\hline Yes & $1.92(1.37-2.68)$ & 0 & $1.96(1.13-3.38)$ \\
\hline \multicolumn{4}{|c|}{ Previous TB treatment history } \\
\hline No & 1.00 & & 1.00 \\
\hline Yes & I $3.9(9.5-20.3)$ & 0 & $11.95(6.78-21.1)$ \\
\hline \multicolumn{4}{|c|}{ History of hospitalization } \\
\hline No & 1.00 & & 1.00 \\
\hline Yes & $6.5 I(4.28-9.91)$ & 0 & $4.14(2.19-7.84)$ \\
\hline \multicolumn{4}{|c|}{ Smear result at the time of diagnosis } \\
\hline Negative & 1.00 & & 1.00 \\
\hline Positive & $1.72(1.25-2.36)$ & 0.001 & $1.95(1.13-3.35)$ \\
\hline \multicolumn{4}{|l|}{ HIV status } \\
\hline NR & 1.00 & & 1.00 \\
\hline $\mathrm{R}$ & $0.63(0.42-0.96)$ & 0.029 & $0.33(0.16-0.66)$ \\
\hline Unknown & $0.72(0.31-1.66)$ & 0.445 & $1.47(0.43-5.01)$ \\
\hline \multicolumn{4}{|c|}{ Diabetes mellitus status of the patient } \\
\hline No + unknown & 1.00 & & 1.00 \\
\hline Yes & $0.45(0.18-1.11)$ & 0.084 & $0.20(0.05-0.83)$ \\
\hline \multicolumn{4}{|c|}{ Disclosure statue of the patient } \\
\hline Disclosed & 1.00 & & 1.00 \\
\hline Did not disclose & $3.72(1.88-7.38)$ & 0 & $5.14(1.84-14.39)$ \\
\hline
\end{tabular}

Abbreviations: AOR, adjusted odds ratio; COR, crude odds ratio; MDR-TB, multidrug-resistant tuberculosis; HIV, human immunodeficiency virus; R, reactive; NR, nonreactive.

HIV has been reported as a major factor, creating a fertile ground for the TB disease. In this study, the TB-HIV co-infection rate was $21 \%$, (cases: $16.2 \%$; controls: $23.1 \%$ ). This finding was comparable with the prevalence estimated for Ethiopia in 2012 (19.7\%). ${ }^{1}$ In the current study, however, HIV-positive patients were less among MDR-TB than drugsusceptible TB. This is probably due to high mortality rate observed among MDR-TB patients. Until the establishment of the first MDR-TB treatment center in Ethiopia in 2009, MDR-TB patients had to face long delays before accessing adequate diagnostic and treatment services, which may explain that HIV-positive MDR-TB patients might have died while on a long waiting list for the treatment. Contrarily, in Europe, HIV was found to be an independent determinant for MDR-TB (OR: 3.52). ${ }^{15}$ A report in South Africa and Burkina Faso $^{11,12}$ indicated that in Africa, no significant association was found between HIV infection and MDR-TB.

In this study, diabetes mellitus was about $70 \%$ less common among cases than controls (AOR: 0.29) But, no significant association was reported from other similar study conducted elsewhere. ${ }^{16}$ To hypothesize on the reasons, it is crucial to report the results of Swedish study investigating the different susceptibility to natural immunity of susceptible versus resistant $M$. tuberculosis strains, and the growth rate of each strain. The results suggest that resistant mutants are likely to be more prone to natural immune defense mechanisms than drug-susceptible ones, even if the host immune system is compromised. ${ }^{17}$ In addition, the commonly distributed type of strain into the community can also explain this finding. However, further broader studies are suggested to confirm this hypothesis.

\section{Conclusion}

The current study identifies that determinants for MDR-TB in the study setting are history of previous TB treatment, failure to disclose TB disease to others, history of hospitalization due to TB, contact with an index case and sputum smear positivity. Whereas, HIV co-infection and diabetes are not associated with MDR-TB.

There is a compelling need to revise the MDR-TB diagnostic and treatment protocol with special emphasis on patients with previous TB treatment, history of contact, hospitalization history for TB management and smear positive cases if universal DST is not possible. Moreover, there is a 
need to enhance TB disease disclosure and comprehensive advocacy effort to reduce social stigma and to sustain the expansion of MDR-TB services in Ethiopia. Implementation of adequate TB infection control practices at health facilities is of paramount importance.

\section{Acknowledgments}

We would like to thank Haramaya University, College of Health and Medical Sciences; Addis Ababa City Administration Health Bureau; St. Peter TB Specialized Hospital and ALERT Medical Center for allowing us to conduct this study.

We would like to disclose that the abstract of this paper was presented at the 45 th World Conference on Lung Health of the International Union against Tuberculosis and Lung Disease (The Union), Barcelona, Spain, October 28, 2014 to November 1, 2014 as a poster presentation. The abstract was published in "Abstract Book" in The International Journal of Tuberculosis and Lung Disease, Vol 18, Number 11 (Abstract Number: PD-1176-01).

\section{Author contributions}

All authors contributed toward data analysis, drafting and critically revising the paper and agree to be accountable for all aspects of the work.

\section{Disclosure}

The authors report no conflicts of interest in this work.

\section{References}

1. Fedreral Minisrty of Health of Ethiopia. Guidelines for Clinical and Programmatic Management of TB, TB/HIV and Leprosy in Ethiopia. 5th ed. Addis Ababa, Ethiopia: Fedreral Minisrty of Health of Ethiopia; 2013.
2. World Health Organization (WHO). Guideline for the Programatic Management of Tuberculosis. Geneva, Switzerland: World Health Organization; 2011.

3. WHO. Fact Sheet for Tuberculosis. Geneva, Switzerland: WHO; 2012.

4. WHO. WHO Report for Global Tuberculosis Control. Geneva, Switzerland: World Health Organization; 2011.

5. FMoHE. Guideline for Prevention of Transmision of Tuberculosis in Health Care Facilities, Gongregate and Community Settings in Ethiopia. Addis Ababa, Ethiopia: FMoHE; 2009.

6. WHO. Global Tuberculosis Report. Geneva, Switzerland: World Health Organization; 2012.

7. WHO. Towards Universal Access to Diagnosis and Treatment of Multidrug-Resistant and Extensively Drug-Resistant Tuberculosis by 2015. Geneva, Switzerland: World Health Organization; 2011.

8. Fedreral Minisrty of Health of Ethiopia. Guide Line for Program and Clinical Management of Drug Resistant Tuberculosis. 1st ed. Addis Ababa, Ethiopia: FMOHE; 2009.

9. Brewer TF, Choi HW, Carlos Seas. Self-Reported Risks for MultipleDrug Resistance among New Tuberculosis Cases: Implications for Drug Susceptibility Screening and Treatment. PLoS One. 2011;6(10). Epub 2011 Oct 14.

10. Casal M, Vanguero M, Rinder H, et al. A case-control study for multidrug-resistant tuberculosis: risk factors in four European countries. Microb Drug Resist. 2005;11(1):62-67.

11. Weyer K, Brand J, Lancaster J, Levin J, van der Walt M. Determinants of multidrug-resistant tuberculosis in South Africa: results from a national survey. S Afr Med J. 2007;97(11):1120-1128.

12. Diande' S, Sangaré L, Kouanda S, et al. Risk factors for multidrugresistant tuberculosis in four centers in Burkina Faso, West Africa. Microb Drug Resist. 2009;15(3):217-221.

13. Marahatta SB, Kaewkungwal J, Ramasoota P, Singhasivanon P. Risk factors of multidrug resistant tuberculosis in central Nepal. Kathmandu Univ Med J (KUMJ). 2010;8(32):392-397.

14. Andrews JR, Shah NS, Weissman D, Moll AP, Friedland G, Gandhi NR. Predictors of multidrug- and extensively drug-resistant tuberculosis in a high HIV prevalence community. PLoS One. 2010;5(12):1-6.

15. Faustini A, Hall AJ, Perucci CA. Risk factors for multidrug resistant tuberculosis in Europe: a systematic review. Thorax. 2006;61(2):158-163.

16. Barroso EC, Salani Mota RM, Santos RO, Oliveira AL, Barroso JB, Rodrigues JL. Risk factors for acquired multidrug-resistant tuberculosis. J Pneumol. 2003;29(2):89-97.

17. Toro JC, Hoffner S, Linde C, Andersson M, Andersson J, Grundstro S. Enhanced susceptibility of multidrug resistant strains of Mycobacterium tuberculosis to granulysin peptides correlates with a reduced fitness phenotype. Microbes Infect. 2006;8(8):1986-1992.
Infection and Drug Resistance

\section{Publish your work in this journal}

Infection and Drug Resistance is an international, peer-reviewed openaccess journal that focuses on the optimal treatment of infection (bacterial, fungal and viral) and the development and institution of preventive strategies to minimize the development and spread of resistance. The journal is specifically concerned with the epidemiology of antibiotic

\section{Dovepress}

resistance and the mechanisms of resistance development and diffusion in both hospitals and the community. The manuscript management system is completely online and includes a very quick and fair peerreview system, which is all easy to use. Visit http://www.dovepress.com/ testimonials.php to read real quotes from published authors. 